\title{
Theory of electrical spin injection: Tunnel contacts as a solution of the conductivity mismatch problem
}

\section{Citation}

Rashba, E. I. 2000. “Theory of Electrical Spin Injection: Tunnel Contacts as a Solution of the Conductivity Mismatch Problem." Physical Review B 62 (24) (December 15): R16267-R16270. doi:10.1103/physrevb.62.r16267.

\section{Published Version}

doi:10.1103/physrevb.62.r16267

\section{Permanent link}

http://nrs.harvard.edu/urn-3:HUL.InstRepos:25872797

\section{Terms of Use}

This article was downloaded from Harvard University's DASH repository, and is made available under the terms and conditions applicable to Other Posted Material, as set forth at http:// nrs.harvard.edu/urn-3:HUL.InstRepos:dash.current.terms-of-use\#LAA

\section{Share Your Story}

The Harvard community has made this article openly available.

Please share how this access benefits you. Submit a story.

Accessibility 


\title{
Theory of electrical spin injection: Tunnel contacts as a solution of the conductivity mismatch problem
}

\author{
E. I. Rashba* \\ Department of Physics, MIT, Cambridge, Massachusetts 02139
}

(Received 11 September 2000)

\begin{abstract}
Theory of electrical spin injection from a ferromagnetic (FM) metal into a normal $(\mathrm{N})$ conductor is presented. We show that tunnel contacts $(\mathrm{T})$ can dramatically increase spin injection and solve the problem of the mismatch in the conductivities of a FM metal and a semiconductor microstructure. We also present explicit expressions for the spin-valve resistance of FM-T-N- and FM-T-N-T-FM-junctions with tunnel contacts at the interfaces and show that the resistance includes both positive and negative contributions (Kapitza resistance and injection conductivity, respectively).
\end{abstract}

\section{INTRODUCTION}

Since the seminal proposal by Datta and Das of a spin transistor ${ }^{1}$ based on spin precession controlled by an external electric field via spin-orbit (SO) coupling, ${ }^{2}$ there exists persistent and growing interest in spin injection into semiconductor microstructures. For a spin transistor to work (i) long spin relaxation time in a semiconductor, (ii) gate voltage control of the SO coupling, and (iii) high spin injection coefficient are needed. Slow relaxation of electron spins in semiconductors has been established by optical experiments. ${ }^{3}$ Modulation of the SO splitting at the Fermi level by gate voltage has been reported for both electrons and holes and for different semiconductor materials. ${ }^{4-8}$ Theory of the gate voltage effect has been developed in much detail. 9,10

However, as distinct from spin injection from a ferromagnetic (FM) source into a paramagnetic metal, very efficient and well documented experimentally, ${ }^{11}$ spin injection from a similar source into a semiconductor ${ }^{12}$ remains a challenging task. After numerous efforts, promising results have been reported recently. ${ }^{13-15}$ Unfortunately, spin polarization measured in Refs. 13 and 14 was only about $1 \%$. Problems with injection from metallic contacts promoted the idea to use a semimagnetic semiconductor as a spin aligner, ${ }^{16}$ and high degree of spin polarization has been achieved in this way. ${ }^{17}$ However, FM metal sources remain an indispensable tool for room temperature devices.

Schmidt et al. ${ }^{18}$ revealed that the basic obstacle for spin injection from a FM metal emitter into a semiconductor originates from the conductivity mismatch between these materials. They have shown, that in a diffusive regime the spin injection coefficient $\gamma$ is $\gamma \propto \sigma_{\mathrm{N}} / \sigma_{\mathrm{F}} \ll 1$, where $\sigma_{\mathrm{N}}$ and $\sigma_{\mathrm{F}}$ are conductivities of the normal (N) (semiconductor) and FM (metallic emitter) contacts, respectively. Their result explains, in a natural way, the striking difference between emission from a FM metal into a paramagnetic metal with $\sigma_{\mathrm{N}} / \sigma_{\mathrm{F}} \gtrsim 1$ and a semiconductor with $\sigma_{\mathrm{N}} / \sigma_{\mathrm{F}} \ll 1$. At first glance, the problem seems insurmountable. However, we show in this paper that insertion of a tunnel contact $(\mathrm{T})$ at a FM-N interface can remedy it. This contact takes control over $\gamma$ and eliminates the conductivity mismatch. For this purpose, tunnel resistance $r_{c}$ does not need to be really large. It should only be larger than competing "effective resistances" making the total contact resistance:

$$
r_{c} \gtrsim L_{\mathrm{F}} / \sigma_{\mathrm{F}}, \quad \min \left\{L_{\mathrm{N}}, w\right\} / \sigma_{\mathrm{N}},
$$

where $L_{\mathrm{F}}$ and $L_{\mathrm{N}}$ are spin diffusion lengths in the FM and $\mathrm{N}$ conductors, respectively, and $w$ is the $\mathrm{N}$ conductor width.

It is our general conclusion that the spin injection coefficient is controlled by the element of a FM-T-N-junction having the largest effective resistance.

Since the dependence of the FM-T-N-T-FM-junction resistance $\mathcal{R}_{j}$ on the mutual polarization of FM electrodes (spin-valve effect) is used for spin injection detection, we have calculated $\mathcal{R}_{j}$. It originates from the current conversion in the junction and includes, side by side with a positive term (Kapitza resistance), a negative term (injection conductivity) originating from spin injection and proportional to $\gamma^{2}$. This term has never appeared in the literature before.

\section{THEORY OF A FM-T-N-JUNCTION}

To make the effect of a tunnel contact most clear, we simplify the problem of a FM-T-N-junction between semiinfinite $\mathrm{FM}(x<0)$ and $\mathrm{N}(x>0)$ conductors as much as possible. We apply the diffusion approximation and suppose that the T contact, at $x=0$, is spin selective, i.e., has different conductivities, $\Sigma_{\uparrow}$ and $\Sigma_{\downarrow}$, for up and down spins, respectively, and there is no spin relaxation in it. Therefore, the problem differs from that considered by van Son et al. ${ }^{19}$ only by the presence of the T contact. Because of some subtleties in calculating the potential distribution near spin emitting contacts, we outline the procedure in some detail.

In the approximation linear in the total current $J$, the currents $j_{\uparrow, \downarrow}(x)$ carried by up- and down-spins can be written in terms of the space derivatives of electrochemical potentials $\zeta_{\uparrow, \downarrow}(x)$,

$$
j_{\uparrow, \downarrow}(x)=\sigma_{\uparrow, \downarrow} \zeta_{\uparrow, \downarrow}^{\prime}(x),
$$

which are related to the nonequilibrium parts $n_{\uparrow, \downarrow}(x)$ of the electron concentrations and to the electrical potential $\varphi_{\mathrm{F}}(x)$ in the FM region by equations 


$$
\zeta_{\uparrow, \downarrow}(x)=\left(e D_{\uparrow, \downarrow} / \sigma_{\uparrow, \downarrow}\right) n_{\uparrow, \downarrow}(x)-\varphi_{\mathrm{F}}(x),
$$

and $D_{\uparrow, \downarrow}$ and $\sigma_{\uparrow, \downarrow}$ are diffusion coefficients and conductivities, respectively, of up- and down-spin electrons. These equations should be supplemented by the equation

$$
n_{\uparrow}(x)+n_{\downarrow}(x)=0,
$$

maintaining the electrical neutrality under the spin injection conditions, and the continuity and charge conservation equations

$$
j_{\uparrow}^{\prime}(x)=e n_{\uparrow}(x) / \tau_{s}^{\mathrm{F}}, \quad J=j_{\uparrow}(x)+j_{\downarrow}(x)=\mathrm{const},
$$

where $\tau_{s}^{F}$ is the spin relaxation time. In the "metallic" approximation, Eq. (4) is equivalent to a Poisson equation and connects transport in both the spin channels.

Let us introduce symmetric in spins variables

$$
\zeta_{\mathrm{F}}(x)=\zeta_{\uparrow}(x)-\zeta_{\downarrow}(x), \quad j_{\mathrm{F}}(x)=j_{\uparrow}(x)-j_{\downarrow}(x) .
$$

In these notations, the standard routine results in a diffusion equation

$$
D_{\mathrm{F}} \zeta_{\mathrm{F}}^{\prime \prime}(x)=\zeta_{\mathrm{F}}(x) / \tau_{s}^{\mathrm{F}}, \quad D_{\mathrm{F}}=\left(\sigma_{\downarrow} D_{\uparrow}+\sigma_{\uparrow} D_{\downarrow}\right) / \sigma_{\mathrm{F}},
$$

where $\sigma_{\mathrm{F}}=\sigma_{\uparrow}+\sigma_{\downarrow}$. The equation for $\varphi_{\mathrm{F}}(x)$,

$$
\varphi_{\mathrm{F}}^{\prime}(x)=\left[\left(D_{\uparrow}-D_{\downarrow}\right) / D_{\mathrm{F}}\right]\left(\sigma_{\uparrow} \sigma_{\downarrow} / \sigma_{\mathrm{F}}^{2}\right) \zeta_{\mathrm{F}}^{\prime}(x)-J / \sigma_{\mathrm{F}},
$$

also follows from Eqs. (2)-(5). Restricting ourselves with zero temperature, $T=0,{ }^{20}$ it is convenient to introduce densities of states at the Fermi level, $\rho_{\uparrow, \downarrow}$, and to apply Einstein relations $e^{2} D_{\uparrow, \downarrow}=\sigma_{\uparrow, \downarrow} / \rho_{\uparrow, \downarrow}$. The identities

$$
\begin{gathered}
e^{2} D_{\mathrm{F}}=\left(\sigma_{\uparrow} \sigma_{\downarrow} / \sigma_{\mathrm{F}}\right)\left(\rho_{\mathrm{F}} / \rho_{\uparrow} \rho_{\downarrow}\right), \\
\left(\rho_{\downarrow} \sigma_{\uparrow}-\rho_{\uparrow} \sigma_{\downarrow}\right) / \rho_{\mathrm{F}} \sigma_{\mathrm{F}}=\left[\left(\Delta \sigma / \sigma_{\mathrm{F}}\right)-\left(\Delta \rho / \rho_{\mathrm{F}}\right)\right] / 2,
\end{gathered}
$$

where $\Delta \sigma=\sigma_{\uparrow}-\sigma_{\downarrow}, \Delta \rho=\rho_{\uparrow}-\rho_{\downarrow}, \rho_{F}=\rho_{\uparrow}+\rho_{\downarrow}$, allow us to rewrite Eq. (8) as

$$
\varphi_{\mathrm{F}}^{\prime}(x)=\left[\left(\Delta \sigma / \sigma_{\mathrm{F}}\right)-\left(\Delta \rho / \rho_{\mathrm{F}}\right)\right] \zeta_{\mathrm{F}}^{\prime}(x) / 2-J / \sigma_{\mathrm{F}} .
$$

It follows from Eqs. (2) and (10) that:

$$
j_{\mathrm{F}}(x)=2\left(\sigma_{\uparrow} \sigma_{\downarrow} / \sigma_{\mathrm{F}}\right) \zeta_{\mathrm{F}}^{\prime}(x)+\left(\Delta \sigma / \sigma_{\mathrm{F}}\right) J .
$$

Equations (7), (10), and (11) make a complete system of bulk equations for the $\mathrm{F}$ region. They also determine

$$
\zeta_{\uparrow}(x)+\zeta_{\downarrow}(x)=-\left[2 \varphi_{\mathrm{F}}(x)+\left(\Delta \rho / \rho_{\mathrm{F}}\right) \zeta_{\mathrm{F}}(x)\right]
$$

and $n_{\uparrow}(x)=\left(\rho_{\uparrow} \rho_{\downarrow} / \rho_{\mathrm{F}}\right) \zeta_{\mathrm{F}}(x)$. Equations for the $\mathrm{N}$ region can be obtained from them by putting $\sigma_{\uparrow}=\sigma_{\downarrow}=\sigma_{\mathrm{N}} / 2, \Delta \rho=\Delta \sigma$ $=0$, and $D_{\mathrm{N}}=D_{\uparrow}=D_{\downarrow}$ :

$$
\begin{gathered}
D_{\mathrm{N}} \zeta_{\mathrm{N}}^{\prime \prime}(x)=\zeta_{\mathrm{N}}(x) / \tau_{s}^{\mathrm{N}}, \quad \varphi_{\mathrm{N}}^{\prime}(x)=-J / \sigma_{\mathrm{N}}, \\
j_{\mathrm{N}}(x)=\sigma_{\mathrm{N}} \zeta_{\mathrm{N}}^{\prime}(x) / 2 .
\end{gathered}
$$

The boundary conditions at $x=0$ follow from the absence of spin relaxation at the interface. The current $j_{\uparrow}(x)$ is continuous at $x=0$ and the condition $j_{\mathrm{F}}(0)=j_{\mathrm{N}}(0)$, according Eqs. (11) and (13), can be rewritten as

$$
\sigma_{\mathrm{N}} \zeta_{\mathrm{N}}^{\prime}(0)-4\left(\sigma_{\uparrow} \sigma_{\downarrow} / \sigma_{\mathrm{F}}\right) \zeta_{\mathrm{F}}^{\prime}(0)=2\left(\Delta \sigma / \sigma_{\mathrm{F}}\right) J
$$

Here $\zeta_{\mathrm{F}}^{\prime}(0)$ and $\zeta_{\mathrm{N}}^{\prime}(0)$ are the values of $\zeta^{\prime}(x)$ at the left and the right sides of the interface, respectively. Low tunnel transparency of the contact supports differences in the potentials $\zeta_{\uparrow, \downarrow}^{\mathrm{F}}$ and $\zeta_{\uparrow, \downarrow}^{\mathrm{N}}$ at the $\mathrm{F}$ and $\mathrm{N}$ sides of it, and makes $\zeta_{\uparrow, \downarrow}(x)$ discontinuous at $x=0 .{ }^{21}$ Similar to Eq. (2), these differences are related to the currents as

$$
j_{\uparrow, \downarrow}(0)=\Sigma_{\uparrow, \downarrow}\left(\zeta_{\uparrow, \downarrow}^{\mathrm{N}}-\zeta_{\uparrow, \downarrow}^{\mathrm{F}}\right),
$$

or, in the symmetric variables of Eq. (6), as

$$
\begin{gathered}
\zeta_{\mathrm{N}}(0)-\zeta_{\mathrm{F}}(0)=-2(\Delta \Sigma / \Sigma) r_{c} J+2 r_{c} j(0), \\
\left(\varphi_{\mathrm{F}}(0)-\varphi_{\mathrm{N}}(0)\right)+\frac{\Delta \rho}{2 \rho_{\mathrm{F}}} \zeta_{\mathrm{F}}(0)=r_{c} J-\frac{\Delta \Sigma}{\Sigma} r_{c} j(0),
\end{gathered}
$$

where Eq. (12) has been taken into account. The current $j(0)=j_{\mathrm{F}}(0)=j_{\mathrm{N}}(0)$ should be found from Eqs. (11) or (13). Here $\Delta \Sigma=\Sigma_{\uparrow}-\Sigma_{\downarrow}, \Sigma=\Sigma_{\uparrow}+\Sigma_{\downarrow}$, and $r_{c}=\Sigma / 4 \Sigma_{\uparrow} \Sigma_{\downarrow}$ is the effective contact resistance.

One important conclusion follows from Eq. (17) immediately: a finite voltage drop at the interface, $V_{\text {if }}=\varphi_{\mathrm{F}}(0)$ $-\varphi_{\mathrm{N}}(0) \propto J$, exists even for $r_{c}=0$ because of $\Delta \rho \neq 0$. This fact is not surprising. Similar discontinuities exist at abrupt $p$ - $n$-junctions ${ }^{22}$ and near current converting surfaces in the theory of the diffusion size effect. ${ }^{23}$ They should also contribute to the giant magnetoresistance. ${ }^{24}$

\section{INJECTION COEFFICIENT}

Solutions of Eqs. (7) and (13) for $\zeta_{\mathrm{F}, \mathrm{N}}(x)$ are exponents decaying with the diffusion lengths $L_{\mathrm{F}}=\left(D_{\mathrm{F}} \tau_{s}^{\mathrm{F}}\right)^{1 / 2}$ and $L_{\mathrm{N}}$ $=\left(D_{\mathrm{N}} \tau_{s}^{\mathrm{N}}\right)^{1 / 2}$. Therefore, $\zeta_{\mathrm{N}}^{\prime}(0)=-\zeta_{\mathrm{N}}(0) / L_{\mathrm{N}}=2 \gamma J / \sigma_{\mathrm{N}}$ and $\zeta_{\mathrm{F}}^{\prime}(0)=\zeta_{\mathrm{F}}(0) / L_{\mathrm{F}}$. Let us define the injection coefficient as $\gamma=j(0) / J$. Eliminating $\zeta_{\mathrm{F}}(0)$ from Eqs. (14) and (16), we get

$$
\gamma=\left[r_{\mathrm{F}}\left(\Delta \sigma / \sigma_{\mathrm{F}}\right)+r_{c}(\Delta \Sigma / \Sigma)\right] / r_{\mathrm{FN}}
$$

where $\quad r_{\mathrm{FN}}=r_{\mathrm{F}}+r_{\mathrm{N}}+r_{c}, \quad r_{\mathrm{F}}=L_{\mathrm{F}} \sigma_{\mathrm{F}} / 4 \sigma_{\uparrow} \sigma_{\downarrow}, \quad$ and $\quad r_{\mathrm{N}}$ $=L_{\mathrm{N}} / \sigma_{\mathrm{N}}$. The equation for $r_{\mathrm{FN}}$ shows that $r_{c}, r_{\mathrm{F}}$, and $r_{\mathrm{N}}$ are connected in series. It follows from Eq. (18) that with $r_{\mathrm{F}} \ll r_{\mathrm{N}}$, the injection coefficient can be large, $\gamma \sim 1$, if and only if $r_{c} \gtrsim r_{\mathrm{N}}$, in agreement with Eq. (1). This criterion is rather soft and is satisfied for narrow tunnel junctions of the atomic scale. Actually, any kind of a spin selective contact with high resistance $r_{c}$ suits this criterion. For $r_{c} \gg r_{\mathrm{N}}, r_{\mathrm{F}}$, the injection coefficient $\gamma \approx \Delta \Sigma / \Sigma_{\mathrm{F}}$. In this regime the contact takes control over $\gamma$ and completely determines it.

\section{SPIN-e.m.f.}

The same FM-T-N-junction can be used for detecting spin accumulation $n_{\infty}$ homogeneously produced in the $\mathrm{N}$ region by some external source by measuring open circuit voltage (floating potential) on a FM electrode. This signal is some kind of photo-e.m.f. and has been successfully used by Johnson $^{25}$ for detecting spins injected into paramagnetic metals, while absence of a similar signal from semiconductor heterostructures ${ }^{26}$ signifies low spin injection level. Derivations similar to the presented above result in a spin-e.m.f. signal 


$$
\varphi_{\mathrm{F}}=2 \gamma\left(e D_{\mathrm{N}} / L_{\mathrm{N}}\right) r_{\mathrm{N}} n_{\infty},
$$

where $\gamma$ is defined by Eq. (18), and $n_{\infty}=n_{\uparrow}(x=\infty)$. Large value of $r_{\mathrm{N}}$, while suppressing spin injection, facilitates large magnitude of $\varphi_{\mathrm{F}}$.

\section{RESISTANCE OF A FM-T-N-JUNCTION}

The voltage drop at the interface, $V_{\text {if }}$, permits one to define the interface resistance $R_{\text {if }}=V_{\text {if }} / J$. Finding $\zeta_{F}(0)$ from Eq. (16) and substituting it into Eq. (17), one gets after some algebra:

$$
\begin{aligned}
R_{\mathrm{if}}(\gamma)= & \Sigma^{-1}+\left[r_{\mathrm{F}}\left(\Delta \rho / \rho_{\mathrm{F}}\right)\left(\Delta \sigma / \sigma_{\mathrm{F}}\right)+r_{c}(\Delta \Sigma / \Sigma)^{2}\right] \\
& -\gamma\left[r_{\mathrm{F}}\left(\Delta \rho / \rho_{\mathrm{F}}\right)+r_{c}(\Delta \Sigma / \Sigma)\right] .
\end{aligned}
$$

The first term in Eq. (20) is an intrinsic property of the interface and does not depend on the presence of nonequilibrium spins, while the last two terms cancel when $L_{\mathrm{N}}, L_{\mathrm{F}}$ $\rightarrow 0$. $R_{\text {if }}-\Sigma^{-1}$ is usually positive but under some conditions, e.g., $\Delta \Sigma=0, \Delta \sigma / \Delta \rho<0$, it is negative.

In addition to $V_{\text {if }}$, there exists a potential drop in the regions about $L_{\mathrm{F}}$ and $L_{\mathrm{N}}$ around the interface which is of the same order of magnitude as $R_{\mathrm{if}}-\Sigma^{-1}$. The total resistance of the junction $R_{j}$ can be found by integrating Eqs. (8) and (13) for $\varphi_{\mathrm{F}}$ and $\varphi_{\mathrm{N}}$ and finding the integration constant from Eq. (17). Subtracting the voltage drop over the nominal resistances of the FM and $\mathrm{N}$ regions from the potential difference between their ends, we get

$$
R_{j}\left(\gamma, r_{\mathrm{FN}}\right)=\Sigma^{-1}+\left[r_{\mathrm{F}}\left(\Delta \sigma / \sigma_{\mathrm{F}}\right)^{2}+r_{c}(\Delta \Sigma / \Sigma)^{2}\right]-\gamma^{2} r_{\mathrm{FN}} .
$$

Two last terms in Eq. (21) originate from nonequilibrium spins and cancel when $L_{\mathrm{N}}, L_{\mathrm{F}} \rightarrow 0$. The second term in Eq. (21) is positive and can be identified as Kapitza resistance originating from the conversion of spin flows. The third term is negative and explicitly related to the spin injection. Therefore, we term it injection conductivity. The sum of both nonequilibrium terms in Eq. (21) is always positive. It is interesting to note that the factor $\Delta \rho / \rho_{\mathrm{F}}$ which is present in $R_{\text {if }}$ cancels from $R_{j}$. Resistances $R_{\text {if }}$ and $R_{j}$ can be measured separately in spin-e.m.f. and spin-valve experiments. For $r_{c}$ $=0$, Eqs. (18) and (21) are equivalent to the results by van Son et al. ${ }^{19}$ hence, the resistance found by them should be identified as $R_{j}$.

\section{SPIN INJECTION INTO A FM-T-N-T-FM-JUNCTION}

General equations derived above are also applicable to a system with two interfaces, two tunnel contacts, and an $\mathrm{N}$ region between them. We attach indices $L$ and $R$ to the parameters of the left and right ferromagnets and tunnel contacts and neglect spin relaxation in $\mathrm{N}$ region since in this case equations simplify and the problem can be solved in terms of the parameters of a single FM-T-N-junction. Writing equations similar to Eqs. (14) and (16) for each contact, taking their sums, and eliminating $\zeta_{\mathrm{F}}^{\mathrm{R}}-\zeta_{\mathrm{F}}^{\mathrm{L}}\left[\zeta_{\mathrm{F}}^{\mathrm{R}}\right.$ and $\zeta_{\mathrm{F}}^{\mathrm{L}}$ being values of $\zeta_{\mathrm{F}}(x)$ at the junction boundaries], one finds injection coefficient $\Gamma=\left(j_{\uparrow}^{\mathrm{N}}-j_{\downarrow}^{\mathrm{N}}\right) / J$ :

$$
\Gamma=\left(r_{\mathrm{FN}}^{\mathrm{L}} \gamma_{L}+r_{\mathrm{NF}}^{\mathrm{R}} \gamma_{\mathrm{R}}\right) / r_{\mathrm{FNF}},
$$

where $r_{\mathrm{FNF}}=r_{\mathrm{N}}^{w}+r_{\mathrm{F}}^{\mathrm{L}}+r_{\mathrm{F}}^{\mathrm{R}}+r_{c}^{\mathrm{L}}+r_{c}^{\mathrm{R}}, r_{\mathrm{N}}^{w}=w / \sigma_{\mathrm{N}}$ is a nominal resistance of the $\mathrm{N}$ region, $w$ is its width, and $\gamma_{\mathrm{L}}$ and $\gamma_{\mathrm{R}}$ can be found from Eq. (18) for L and R interfaces. Similar to Eq. (18), injection is controlled by the larger of the resistances $r_{\mathrm{F}}^{\mathrm{L}, \mathrm{R}}$ and $r_{c}^{\mathrm{L}, \mathrm{R}}$. To achieve a large $\gamma$ value it is enough to have only one tunnel contact, either the left or the right one. The second contact is only needed for detecting spin injection by the spin-valve effect. Even in a completely antisymmetric system, $\Delta \sigma_{\mathrm{L}} / \sigma_{\mathrm{L}}=-\Delta \sigma_{\mathrm{R}} / \sigma_{\mathrm{R}}, \Delta \Sigma_{\mathrm{L}} / \Sigma_{\mathrm{L}}=-\Delta \Sigma_{\mathrm{R}} / \Sigma_{\mathrm{R}}, r_{c}^{\mathrm{L}}$ $=r_{c}^{\mathrm{R}}$, nonequilibrium spins are present in the $\mathrm{N}$ region. That is, for $r_{c}^{\mathrm{L}}=r_{c}^{\mathrm{R}}=0$, their concentration equals $n_{\uparrow}^{\mathrm{N}}(x)=$ $-\left(\sigma_{\mathrm{N}} / 2 D_{\mathrm{N}}\right)\left(\Delta \sigma_{\mathrm{L}} / \sigma_{\mathrm{F}}\right) r_{\mathrm{F}} J=\mathrm{const}$, and the result $\Gamma=0$ following from Eq. (22) is tantamount to the absence of diffusion currents in the N-region. Nonequilibrium spins in it can be detected by spin-e.m.f.

\section{RESISTANCE OF A FM-T-N-T-FM-JUNCTION}

Similar to a FM-N-junction, $\varphi(x)$ shows abrupt change at both interfaces and gradual change near them at the scale of $L_{\mathrm{F}}$. Interfacial resistances $\mathcal{R}_{\mathrm{if}}^{\mathrm{L}, \mathrm{R}}$ are similar to Eq. (20):

$$
\mathcal{R}_{\text {if }}^{\mathrm{L}, \mathrm{R}}=R_{\text {if }}^{\mathrm{L}, \mathrm{R}}\left(\Gamma, r_{\mathrm{FNF}}\right),
$$

i.e., they can be found from Eq. (21), however, with $\Gamma$ instead of $\gamma$ and $r_{\mathrm{FNF}}$ instead of $r_{\mathrm{FM}}$. The junction resistance $\mathcal{R}_{j}$ can be written in a similar way in terms of $R_{j}$ :

$$
\mathcal{R}_{j}=r_{\mathrm{N}}^{w}+R_{j}^{\mathrm{L}}\left(\Gamma, r_{\mathrm{FNF}}\right)+R_{j}^{\mathrm{R}}\left(\Gamma, r_{\mathrm{FNF}}\right),
$$

i.e., it can be found from Eq. (21) by plugging into it the parameters of both contacts and changing $\gamma \rightarrow \Gamma$ and $r_{\mathrm{FN}}$ $\rightarrow r_{\mathrm{FNF}}$. Therefore, $\mathcal{R}_{j}$ also includes the Kapitza resistance and injection conductivity. The nonequilibrium part of $\mathcal{R}_{j}$ is always positive, but the explicit equation proving this fact is somewhat lengthy.

Let us mention that Eqs. (23) and (24) for resistances, as well as Eqs. (20) and (21), include only products or squares of the differences $\Delta \sigma, \Delta \Sigma$ and $\Delta \rho$, while the equations for potentials [like Eqs. (14), (16), and (17)] include them in the first power.

Detection of spin injection by the spin-valve effect is based on the change in $\mathcal{R}_{j}$ when the magnetization direction of one of the two identical FM-electrodes is reversed $\left(\Delta \sigma_{\mathrm{R}}\right.$ $\left.\rightarrow-\Delta \sigma_{\mathrm{R}}, \Delta \Sigma_{\mathrm{R}} \rightarrow-\Delta \Sigma_{\mathrm{R}}\right)$. It comes exclusively from the injection conductivity, $\Delta \mathcal{R}_{j}=r_{\mathrm{FNF}}\left(\Gamma_{\uparrow \uparrow}^{2}-\Gamma_{\uparrow \downarrow}^{2}\right)$, and equals:

$$
\Delta \mathcal{R}_{j}=\gamma_{\mathrm{L}} \gamma_{\mathrm{R}}\left(4 r_{\mathrm{FN}}^{\mathrm{L}} r_{\mathrm{NF}}^{\mathrm{R}} / r_{\mathrm{FNF}}\right)
$$

The resistance $\mathcal{R}_{j}$, Eq. (24), remains finite for a completely antisymmetric system even for $|\Delta \sigma| \ll \sigma_{\mathrm{F}}$. In the absence of spin relaxation in the $\mathrm{N}$ region and at both contacts, no conductivity through a junction could be expected at the first glance. However, it exists and its mechanism is as follows. Because $\Gamma_{\uparrow \downarrow}=0$, the currents $j_{\uparrow}^{\mathrm{N}}$ and $j_{\downarrow}^{\mathrm{N}}$ in the $\mathrm{N}$ region are driven only by the electric field and are equal exactly, $j_{\uparrow}^{\mathrm{N}}=j_{\downarrow}^{\mathrm{N}}=J / 2$. In the FM regions, the currents of the minority spins are driven mostly by diffusion. Therefore, the concentrations of nonequilibrium spins near the interfaces equal $n$ $\approx\left(L_{\mathrm{F}} / 2 e D_{\mathrm{F}}\right) J$, and the only restriction on the diffusion current comes from the condition that the total concentration of minority carriers is positive, i.e., $n_{\min }^{0} \pm n>0$, where $n_{\min }^{0}$ is 
the equilibrium concentration of minority carriers. Hence, the ohmic conductivity of a FM-T-N-T-FM-junction remains finite even when $|\Delta \sigma| / \sigma_{\mathrm{F}} \rightarrow 0$, but the ohmic region becomes narrower and disappears completely for $|\Delta \sigma|=\sigma_{\mathrm{F}}$. Nonlinear conductivity is outside the scope of this paper.

For $r_{c}^{\mathrm{L}}=r_{c}^{\mathrm{R}}=0$, Eq. (22) for $\Gamma$ is equivalent to the result by Schmidt et al. ${ }^{18}$ However, Eqs. (24) and (25) differ from the equations for ohmic resistance of Refs. 18 and 26. Because the derivation procedure has not been specified there, the origin of the discrepancy is unclear.

\section{DISCUSSION}

The above theory suggests that tunnel contacts obeying criterion equation (1) should provide a tremendous increase in spin polarization of the currents injected electrically from a FM metal into a semiconductor. Our conclusion is based on the assumption that spin conductivity ratio $\Delta \Sigma / \Sigma$ is large for tunnel contacts. In fact, Alvorado ${ }^{27}$ has shown that spin polarization is large for narrow barriers and can reach about $50 \%$. Different types of tunnel contacts have been success- fully used, e.g., STM tips in vacuum ${ }^{27}$ and in air, ${ }^{28}$ Schottky barriers, ${ }^{29,30}$ and resonant double barriers. ${ }^{31}$ Therefore, inclusion of appropriate barriers into a circuit should be a soluble problem. One should also bear in mind, that it is not the ballistic transport, but the ability of tunnel contacts to support a considerable difference in electrochemical potentials under the conditions of slow spin relaxation, which is important for efficient spin injection. Therefore, different contacts combining small spin diffusivity with low spin relaxation rate should possess similar properties.

In conclusion, we (i) have shown that tunnel contacts can solve the problem of the electrical spin injection from a ferromagnetic metal into a semiconductor, and (ii) have derived explicit expressions for the spin injection coefficient, spinvalve effect, and spin-e.m.f.

\section{ACKNOWLEDGMENT}

I am grateful to Dr. Alexander Efros for useful discussions.
*Electronic address: erashba@hotmail.com

${ }^{1}$ S. Datta and B. Das, Appl. Phys. Lett. 56, 665 (1990).

${ }^{2}$ E. I. Rashba, Sov. Phys. Solid State 2, 1109 (1960); E. I. Rashba and V. I. Sheka, in Landau Level Spectroscopy, edited by G. Landwehr and E. I. Rashba (North-Holland, Amsterdam, 1991), Vol. 1, p. 131.

${ }^{3}$ Optical Orientation, edited by F. Meier and B. P. Zakharchenya (North-Holland, Amsterdam, 1984); J. M. Kikkawa and D. D. Awschalom, Phys. Rev. Lett. 80, 4313 (1998); D. Hägele et al., Appl. Phys. Lett. 73, 1580 (1998).

${ }^{4}$ M. Schultz et al., Semicond. Sci. Technol. 11, 1168 (1996).

${ }^{5}$ J. Nitta et al., Phys. Rev. Lett. 78, 1335 (1997); J. P. Lu et al., ibid. 81, 1282 (1998).

${ }^{6}$ G. Engels et al., Phys. Rev. B 55, R1958 (1997); J. P. Heida et al., ibid. 57, 11911 (1998); C.-M. Hu et al., ibid. 60, 7736 (1999).

${ }^{7}$ Y. Sato et al., Physica B 272, 114 (1999); S. Sasa et al., ibid. 272, 149 (1999).

${ }^{8}$ D. Grundler, Phys. Rev. Lett. 84, 6074 (2000).

${ }^{9}$ G. Lommer, F. Malcher, and U. Rössler, Phys. Rev. Lett. 60, 728 (1988); P. V. Santos and M. Cardona, ibid. 72, 432 (1994).

${ }^{10}$ E. A. de Andrada e Silva, G. C. La Rocca, and F. Bassani, Phys. Rev. B 55, 16293 (1997); P. Pfeffer and W. Zawadzki, ibid. 59, R5312 (1999); R. Winkler, ibid. 62, 4245 (2000).

${ }^{11}$ P. M. Todrow and M. Meservey, Phys. Rev. Lett. 26, 192 (1971); M. Johnson and R. H. Silsbee, ibid. 55, 1790 (1985).

${ }^{12}$ A. G. Aronov and G. E. Pikus, Sov. Phys. Semicond. 10, 698 (1976).
${ }^{13}$ P. Hammer et al., Phys. Rev. Lett. 83, 203 (1999).

${ }^{14}$ S. Gardelis et al., Phys. Rev. B 60, 7764 (1999).

${ }^{15} \mathrm{G}$. Meier, D. Grundler, T. Matsuyama, and U. Merkt, Abstracts of the Symp. on Spin-Electronics (Halle, July, 2000).

${ }^{16}$ M. Oestreich et al., Appl. Phys. Lett. 74, 1251 (1999).

${ }^{17}$ R. Fiederling et al., Nature (London) 402, 787 (1999); Y. Ohno et al., ibid. 402, 790 (1999); B. T. Jonker et al., Phys. Rev. B 62, R4790 (2000).

${ }^{18}$ G. Schmidt, D. Ferrand, L. W. Mollenkamp, A. T. Filip, and B. J. van Wees, Phys. Rev. B 62, R4790 (2000).

${ }^{19}$ P. C. van Son, H. Kempen, and P. Wyder, Phys. Rev. Lett. 58, 227 (1987).

${ }^{20}$ This restriction does not influence the basic results.

${ }^{21}$ Criterion (1) is equivalent to $t \leqq\left(\tau_{p} / \tau_{s}\right)^{1 / 2}$, where $t$ is tunnel transparency, and $\tau_{p}$ and $\tau_{s}$ are momentum and spin relaxation times. It ensures discontinuity of $\zeta(x)$ at $x=0$.

${ }^{22}$ W. Shockley, Bell Syst. Tech. J. 28, 435 (1949).

${ }^{23}$ V. Ya. Kravchenko and E. I. Rashba, Sov. Phys. JETP 29, 918 (1969).

${ }^{24}$ G. A. Prinz, Science 282, 1660 (1998).

${ }^{25}$ M. Johnson, Phys. Rev. Lett. 70, 2142 (1993).

${ }^{26}$ A. T. Filip et al., Phys. Rev. B 62, 9996 (2000).

${ }^{27}$ S. F. Alvorado, Phys. Rev. Lett. 75, 513 (1995).

${ }^{28}$ C. J. Hill et al., cond-mat/0010058 (4 October 2000).

${ }^{29}$ D. J. Monsma et al., Science 281, 407 (1998).

${ }^{30}$ V. Delmouly, A. Bournel, G. Tremblay, and P. Hesto, Abstracts of the Symp. on Spin-Electronics (Halle, July, 2000).

${ }^{31}$ H. Ohno, Science 281, 951 (1998). 